\section{Contralateral auditory change masking}

\author{
R. E, PASTORE, L. B. HARRIS, \\ and C. J. FRIEDMAN \\ State University of New York \\ Binghamton, New York 13901
}

Pastore and MacLatchy (1975) have demonstrated that a postsignal decrease in an otherwise continuous masking noise has a backward masking effect relative to the same stationary noise. Their basic notation was that any changes in the parameters of the masker that are temporally in proximity to the signal can serve to increase the effective variability of the noise, thereby reducing the functional signal-to-noise ratio. An alternative interpretation of these results is that subjects had been detecting off-frequency signal transients which now are masked by a spread in energy associated with noise offset in the same earphone. These two hypotheses can be differentiated by presenting the noise change in a waveform physically separate from that containing the signal with its possible off-frequency transients. This paper describes two experiments designed to provide such a test. The dichotic presentation of monaural waveforms cannot involve any significant interaction of the physical waveforms; any interactions must have a sensory or perceptual basis. Our two experiments attempted to find backward masking and forward masking effects by employing changes in the nature (but not intensity) of the noise in the nonsignal earphone.

\section{EXPERIMENT 1: BACKWARD MASKING}

Backward masking most probably has a major central component that may involve uncertainty effects about signal or noise parameters. For instance, the use of a contralateral cue that provides the subject with exact information about when the potential signal can occur will significantly reduce backward masking (Puleo \& Pastore, 1980), implying that backward masking involves a high degree of uncertainty about when to most effectively listen for the signal. Such uncertainty represents an identifiable difference between an actual subject and an ideal signal-known-exactly (SKE) observer model (Tanner, 1961). The hypothesis of change-induced noise variability would represent another type of such potential uncertainty, therefore resulting in further deviation from an SKE model and, consequently, re-

This research was supported by Grant NS 10995 from NINCDS to the first author. Reprint requests should be sent to the Psychoacoustics Laboratory, Department of Psychology, SUNY, Binghamton, New York 13901 . duced signal detectability relative to a less uncertain situation.

\section{Method}

Subjects. Two male and two female undergraduates with no known hearing deficits were employed as professional subjects. All had previous experience in auditory detection tasks.

Procedure. The signal was a $10-\mathrm{msec}$ segment of monaural $1,000-\mathrm{Hz}$ sinusoid presented in a continuous $60-\mathrm{dBA}$ Gaussian noise (filtered between 315 and $3,150 \mathrm{~Hz}$ ). The nonsignal earphone always contained the identical noise from either the inverted $(-1$ correlation) or the noninverted $(+1$ correlation) output of a single operational amplifier. The various conditions in the study differed solely in terms of noise in the nonsignal earphone. In the control conditions, this noise was continuous in its +1 or -1 form. In the experimental conditions, the contralateral noise was inverted $\mathrm{t}$ msec following signal offset for a duration of $\mathrm{d} \mathrm{msec}$ $(\mathrm{t}=2,5,10,20,40,80,160 ; \mathrm{d}=10,20,40,80,160)$. The change was created by simultaneously gating one noise form off and the other on; all stimuli were gated with 1-msec rise/fall times.

Thresholds ( $71.6 \%$ correct) were determined with a Levitt (1971) Up-Down single-interval adaptive procedure with a $2: 1$ rule and a 2-dB step size for signal intensity. Each data point is based upon two consecutive blocks of 120 trials for which both the standard deviation within a sequence and the total range of the four sequence means were less than $4 \mathrm{~dB}$ for the given subjects. Since the signal was monaural, threshold signal-to-noise ratios $(10 \mathrm{log}$ $\mathrm{E} / \mathrm{N}_{\mathrm{o}}$ ) are based upon monaural noise and signal.

\section{Results and Discussion}

The mean signal-to-noise ratios were 11.8 and 12.2 (SDs of 1.3 and 1.1 ) for the +1 and -1 control condition thresholds. Therefore, there was no difference in the masking effectiveness of the two contralateral noise conditions. Masking in the experimental conditions is estimated relative to the mean control signalto-noise ratio of 12.0. The mean amount of masking (with a range of $1 \mathrm{SD}$ on each side of that mean) for the 20-msec duration noise changes are shown in Figure 1 . These data are typical of all masker durations. There were no systematic or significant differences between the two types of noise correlation changes.

Plotted in Figure 2 are the mean amounts of masking combined across the two directions of noise correlation change for each onset delay (abscissa) and duration (symbols) of the masker. These data do not exhibit any significant trend as a function of masker duration (across symbols), but do exhibit a typical temporal backward masking function. These results are consistent with our original change-induced noise variance hypothesis. A postsignal change in the interaural correlation of the masking noise caused a small (approximately $5 \mathrm{~dB}$ ) masking effect on a monaural signal presented to the earphone not physically containing the change. The off-frequency transient hypothesis cannot easily explain these results.

Interaural phase alone did not significantly alter the monaural masking effectiveness of the noise. Phase does alter the lateralized perception of the noise 


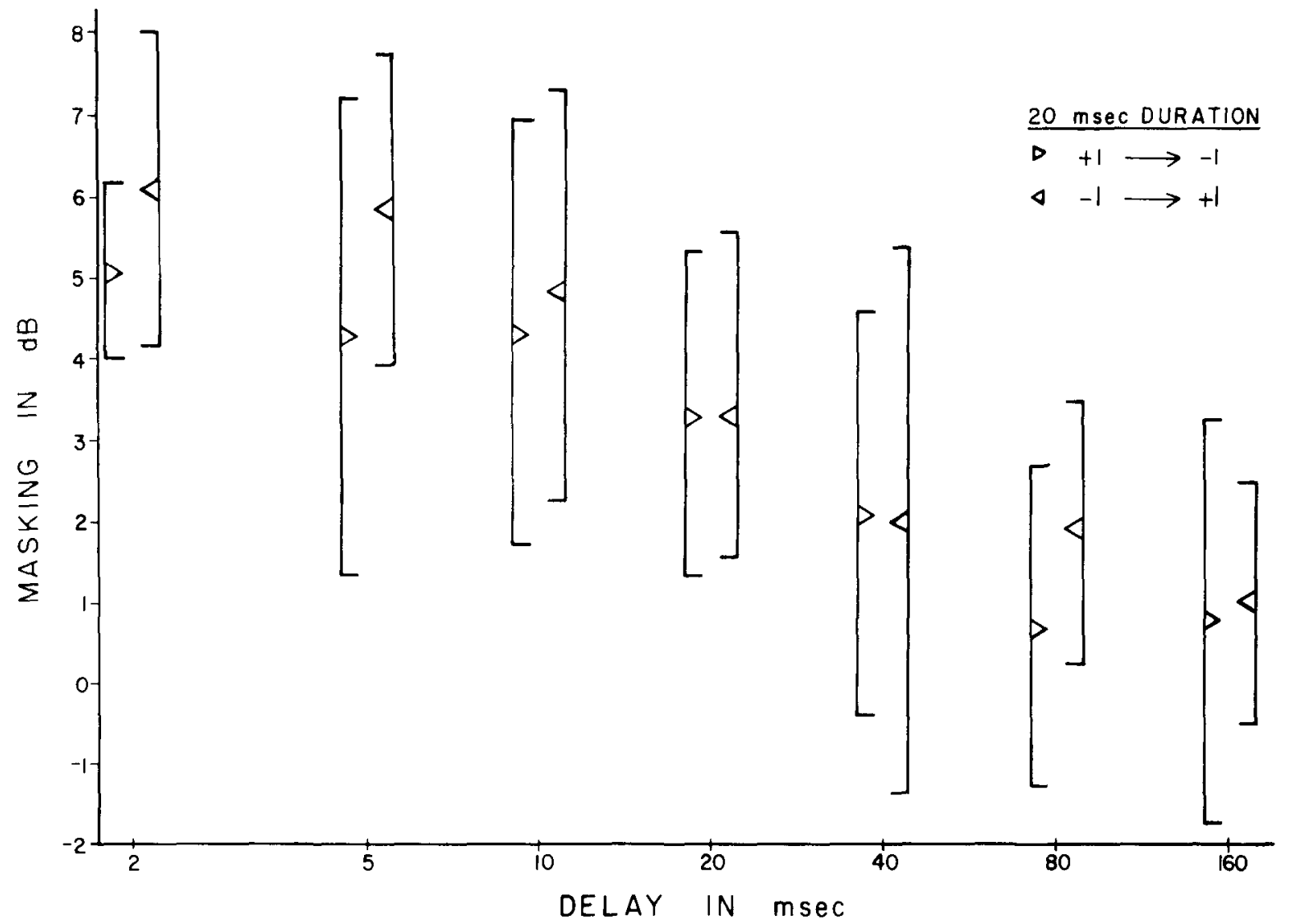

Figure 1. The mean amount of masking in decibels with a range of $1 \mathrm{SD}$ in each side as a function of delay in milliseconds after the signal and before the noise inversion for the 20-msec duration masker in Experiment 1.

(Jeffress, Blodgett, Sandel, \& Wood, 1956). Based upon a signal detection analysis (Egan \& Clarke, 1966), one might conjecture that subjects compare the observed waveform (containing the signal, noise, and noise change) with some stored representation of the parameters of the noise alone (or noise plus noise

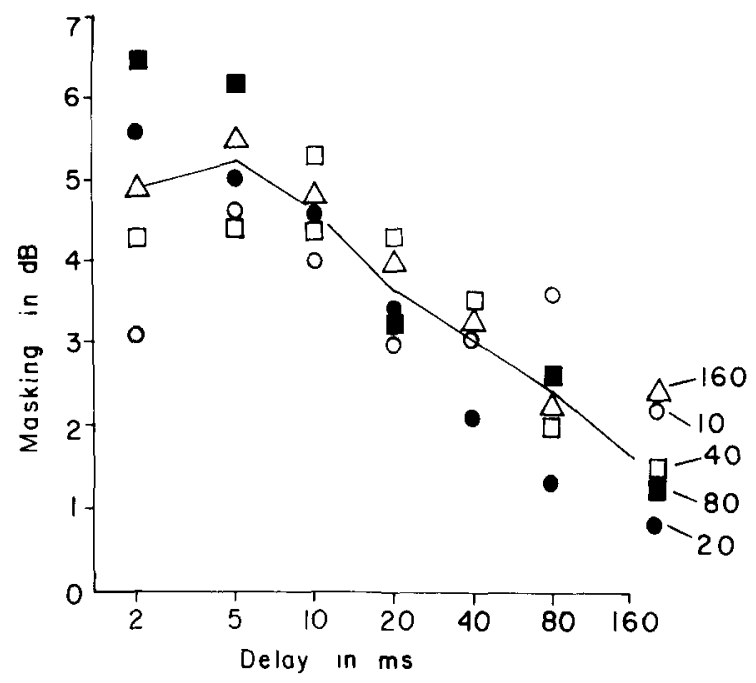

Figure 2. The mean amount of masking in decibels plotted as a function of the delay in milliseconds for both directions of masker change combined for each duration of the masker in Experiment 1. change); that representation probably involves a heavy weighting of noise parameters in temporal proximity to the observation interval. Alternatively, the change might interfere with the storage or processing of information about the potential signal. These hypotheses would predict an effective signalto-noise ratio that is smaller than (or less reliable than) that expected without the noise change. If either of these hypotheses is accurate, then the change will have increased the uncertainty in the detection task. We conjecture that such stimulus change effects are one factor in more conventional backward masking situations and, quite possibly, in forward masking situations.

\section{EXPERIMENT 2: FORWARD MASKING ${ }^{1}$}

If one of the noise variance hypotheses is correct, then it is quite possible that similar contralateralchange-masking effects exist for a forward masking paradigm. If we do not find such forward masking effects, we will have to modify our hypotheses to include only the postsignal noise environment.

\section{Method}

Subjects. There were one male and three female undergraduate subjects, all with no known hearing deficits and all with prior 


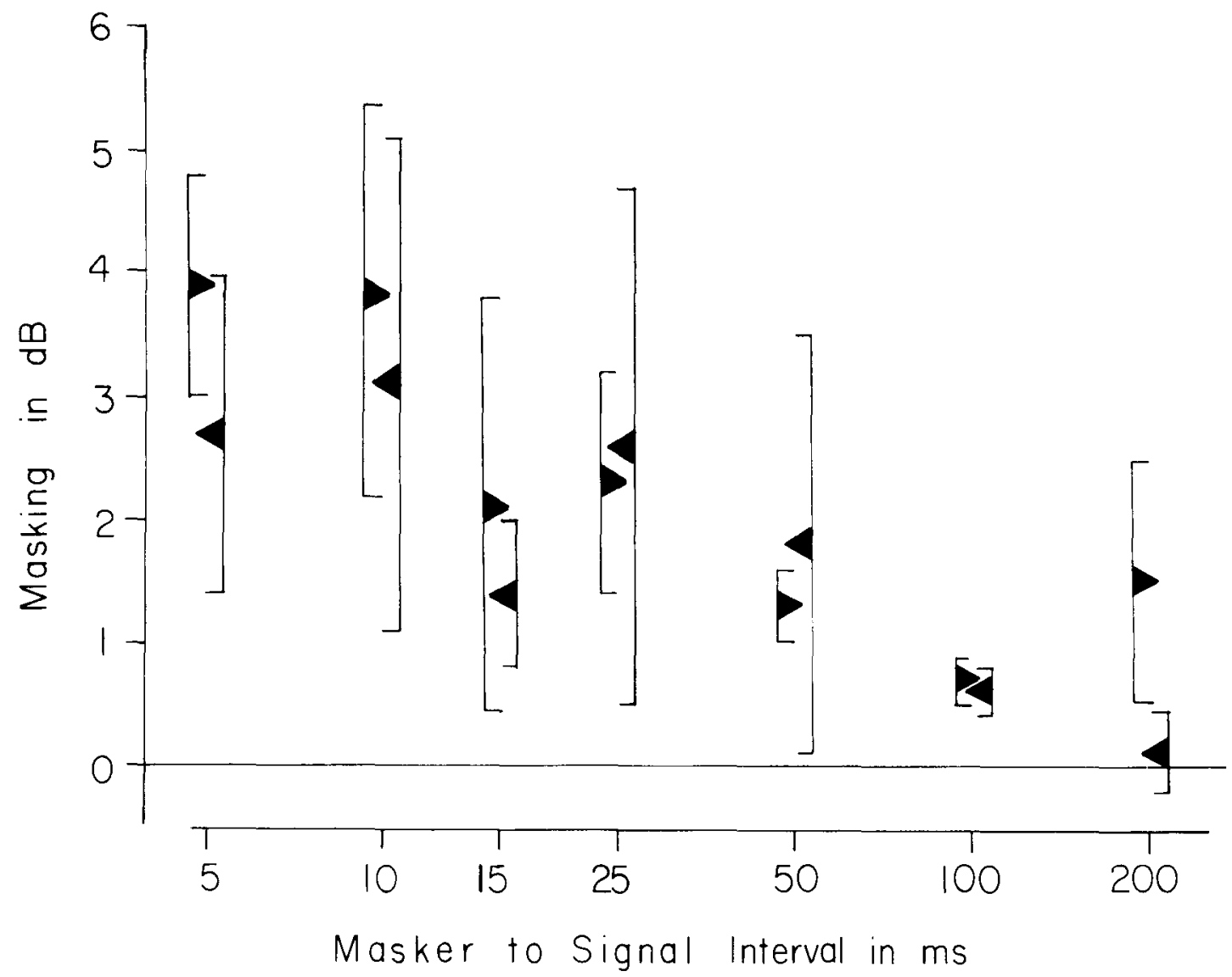

Figure 3. The average amount of masking in decibels with a range of 1 SD on each side as a function of delay between masker onset and the signal in milliseconds for Experiment 2.

experience in the laboratory. (One female subject also was employed in the first experiment.)

Procedure. The stimulus was a $15-\mathrm{msec}$ segment of a monaural $1,000-\mathrm{Hz}$ sinusoid gated at positive waveform zero-crossings and then filtered. The masking noise conditions were identical to those described for Experiment 1 except that the noise was not filtered, and the change in noise phase occurred $t \mathrm{msec}$ before the observation interval $(t=5,10,15,25,50,100,200)$, always returning to its prechange condition $1,000 \mathrm{msec}$ after the observation interval. At each delay, $t$, we generated a three-point psychometric function ( $\log d^{\prime}$ vs. dB) for each observer and condition after asymptotic performance had been achieved. Each data point is based upon five blocks of 100 tasks. The amount of change masking is measured in terms of the additional signal intensity for $\mathrm{d}^{\prime}=1.0$ performance relative to the control conditions.

\section{Results and Discussion}

Performance under the two control conditions again was equivalent. Figure 3 shows the average amount of masking (with range of 1 SD on each side of mean) for each type of presignal change $(+1 /-1$ and $-1 /+1)$ at each value of $t$. These data exhibit a small, although significant, forward masking effect that decreases with increasing $t$ for both types of presignal change. There is no significant difference between types of change. While the magnitudes of these forward masking effects are smaller than those found for backward masking (Figure 2), we are hesitant to draw any inferences from such a comparison because of differences in the experimental procedures employed.

\section{GENERAL DISCUSSION}

It is clear from these data that the detection of a signal is more difficult when it is presented in a nonstationary environment, and that its decreased detection cannot be attributed to the direct interaction of offfrequency energy associated with the gating of the signal and the noise. Our results indicate that this can be the case even when the nonstationarity is a change in the noise presented to an earphone that never contains the signal. Therefore, this change masking must involve a level of processing that is more central than the cochlea.

Signal detection theory models assume that the subject has knowledge of the variability of the noise distribution and the mean effects of adding signal to the noise. In monitoring a potential signal, the subject estimates a decision variable based upon the ratio of the likelihood of a signal relative to a nonsignal event. The likelihood ratio must be based upon the subject's knowledge of the parameters of the under- 
lying distributions. With such an analysis, it can be shown that the ability of the subject to discriminate signal events from nonsignal events is a function of signal-to-noise ratio (Egan \& Clarke, 1966). Nonstationarity in the masker can be expressed as an increase in noise variability, and therefore, with constant signal intensity, as a decrease in "effective" signal-to-noise ratio. Our finding of both forward and backward masking change effects implies that the estimation of noise parameters is based, at least in part, upon a sampling of the noise both immediately before and immediately after the potential signal, and that such sampling depends less upon temporally more distant samples of the noise. This pattern of results is consistent with models of signal processing based upon the weighted integration of stimulus information in time (e.g., Penner, 1979), although clearly more than a weighted summation of energy is involved. Finally, in the context of a signal detection analysis, change masking effects most probably are one contributing factor to the often-reported finding that maskers gated with the signal exhibit greater masking than analogous maskers that are continuous (e.g., Green, 1969).

\section{REFERENCES}

Egan, J. P., \& Clarke, F. R. Psychophysics and signal detection. In J. B. Sidowski (Ed.), Experimental methods and instrumentation in psychology. New York: McGraw-Hill, 1966.
Green, D. M. Masking with continuous and pulsed sinusoids. Journal of the Acoustical Society of America, 1969, 46, 939-946.

Jeffress, L. A., Blodgett, H. C., Sandel, T. T., \& Wood, C. L., III. Masking of tonal signals. Journal of the Acoustical Society of America, 1956, 28, 416-426.

LevitT, H. Transformed up-down methods in psychoacoustics. Journal of the Acoustical Society of America, 1971, 49, 467-477.

Pastore, R. E., \& MacLatchy, C. S. Some nonmasking auditory postsignal effects. Perception \& Psychophysics, 1975, 17, 455-459.

Penner, M. J. Forward masking with equal-energy maskers. Journal of the Acoustical Society of America, 1979, 66, 1719-1724.

Puleo, J. S., \& Pastore, R. E. Contralateral cueing effects in backward masking. Journal of the Acoustical Society of America, $1980,67,947-951$.

TANNER, W. P., JR. Physiological implications of psychophysical data. Annals of the New York Academy of Sciences, 1961, 89, 752-765.

\section{NOTE}

1. The "second" experiment actually was run some time before the "first"; at that time, our laboratory was not employing adaptive psychophysical procedure. The change from $15-\mathrm{msec}$ signals in the forward masking experiment to $10-\mathrm{msec}$ signals in the backward masking experiment was based upon the untested belief that the briefer stimulus would he more susceptible to change masking effects.
(Received for publication March 10, 1980; revision accepted August 28, 1980.) 